\title{
Kulturelle Unterschiede in der Aktualisierung kommunikativer Gattungen ${ }^{1}$
}

\author{
Susanne Günthner
}

\section{Einleitung}

Arbeiten der Interkulturellen Kommunikationsforschung verdeutlichen, daß »Fremdheit « keine Eigenschaft bzw. kein objektives Verhältnis zweier Personen oder Gruppen zueinander ist, sondern Resultat einer interaktiven Erzeugung und Zuschreibung (Hahn 1994). Folglich stellen sich die Fragen: Wie wird »Fremdheit « interaktiv erzeugt? Wie werden Interaktionssituationen $\mathrm{zu}$ »interkulturellen« Begegnungen? Worin unterscheiden sich interkulturelle Kommunikationssituationen von intrakulturellen? (Günthner 1999).

Wie Alfred Schütz (1972) in seinem mittlerweile als Klassiker geltenden Aufsatz »Der Fremde« ausführt, machen wir in einer interaktiven Begegnung dann die Erfahrung von Fremdheit, wenn unsere alltäglichen Interpretations- und Handlungsmuster in Frage gestellt werden und scheitern bzw. wenn unsere bislang als unhinterfragt und selbstverständlich angenommenen Interpretationsschemata nicht mehr länger als ein System erprobten Rezeptwissens fungieren. Die Wissensbestände, die in früheren Kommunikationssituationen zur Interpretation so- zialer Wirklichkeit genügten und sowohl als Handlungsanweisungen als auch als Auslegungsschemata fungierten, erweisen sich als für die neue Situation nur noch bedingt brauchbar. Folglich sind

»die Kultur- und Zivilisationsmuster der Gruppe, welcher sich der Fremde nähert, [...] für ihn kein Schutz, sondern ein Feld des Abenteuers, keine Selbstverständlichkeit, sondern ein fragwürdiges Untersuchungsthema, kein Mittel, um problematische Situationen $\mathrm{zu}$ analysieren, sondern eine problematische Situation selbst und eine, die hart zu meistern ist « (Schütz 1972: 67).

Solche Situationen, in denen wir an die Grenzen unserer als selbstverständlich angenommenen Interpretationsschemata stoßen, führen zu Prozessen der Kategorisierung in »wir « und »sie« und damit zur Konstruktion des »Eigenen « und des »Anderen« bzw. »Fremden« (Schütz 1972, Hahn 1994, Günthner 1999).

Einen wesentlichen Bereich der kommunikativen Praxis, der zur Konstitution von Fremdheit und damit zur Aktualisierung von Interkulturalität beitragen kann, stellt die Orientierung der Interagierenden an kulturell divergierenden rhetorischen Traditionen mit ihren Repertoires an kommunikativen Gattungen dar. ${ }^{2}$ Kul-

1 Peter Auer, Thomas Luckmann und Rainer Rothenhäusler danke ich für ihre Kommentare zu einer früheren Version des Artikels. Es handelt sich hierbei um eine überarbeitete Version des Beitrags, der erschienen ist in: Beiträge zur Fremdsprachenvermittlung aus dem Sprachlehrinstitut der Universität Konstanz 1 (2000).

2 Zum Konzept der kommunikativen Gattungen siehe Luckmann 1988, Bergmann/ Luckmann 1995, Günthner/Knoblauch 1994, 1995 sowie Günthner 1993, 1995). 
turell unterschiedliche Repertoires an kommunikativen Gattungen, divergierende Konventionen scheinbar gleicher Gattungen, unterschiedliche stilistische Bewertungen einer bestimmten Gattung etc. führen immer wieder zu Problemen in Kommunikationssituationen zwischen Angehörigen verschiedener Kulturen bzw. aktualisieren »Fremdheit« (Günthner 1993, Günthner/Luckmann im Druck 2000, 2001).

Ziel dieses Beitrags ist es, die Relevanz der anthropologisch-linguistischen und wissenssoziologischen Gattungsforschung für die Erforschung interkultureller Kommunikationsforschung wie auch für die Deutsch als Fremdsprache-Forschung aufzuzeigen. Zunächst soll das Konzept der kommunikativen Gattungen vorgestellt werden. Im Anschluß daran werde ich anhand konkreter Beispiele interkultureller Begegnungen mögliche Fragestellungen und Konsequenzen für die Erforschung interkultureller Kommunikationssituationen aufzeigen.

\section{Das Konzept der kommunikativen Gattungen}

Das in der Kulturanthropologie, Wissenssoziologie und Gesprächsanalyse verwendete Konzept der »kommunikativen Gattung « knüpft an die in den 20er und 30er Jahren entstandenen Arbeiten Bachtins und Vološinovs an. Sprache ist für Vološinov (1929/75) und Bachtin (1979/ 86) eng mit der sozialen Wirklichkeit verknüpft, da sie in sozialen Situationen Verwendung findet. Sie hat ihren eigentlichen Sitz in der kommunikativen Inter- aktion, und sie wird in Form konkreter Äußerungen in verschiedenartigen Aktivitäten realisiert (Bachtin 1979/86). Zur Realisierung bedienen sich Sprecher/innen interaktionssteuernder Redegattungen, deren Wahl von sozialen Strukturen geprägt ist. Für Bachtin (1979/86: 78 f.) besteht Sprechen somit hauptsächlich in der Wahl einer Redegattung. Gattungen sind als historisch und kulturell spezifische, gesellschaftlich verfestigte und formalisierte Lösungen kommunikativer Probleme $\mathrm{zu}$ betrachten. Ihre Funktion besteht in der Bewältigung, Vermittlung und Tradierung intersubjektiver Erfahrungen der Lebenswelt (Luckmann 1988: 283). Von »spontanen « kommunikativen Vorgängen unterscheiden sie sich dadurch, daß die Interagierenden sich in einer voraussagbaren Typik an vorgefertigten Mustern ausrichten. ${ }^{1}$ So gibt es beispielsweise in unserer Kultur verfestigte, routinisierte Formen von universitären Vorlesungen. Im Laufe des Studiums lernen wir diese universitäre Gattung kennen, kennen ihre Regeln und ihren typischen Verlauf. ${ }^{2}$

Wir orientieren uns an diesem Gattungswissen, das nicht nur bestimmte Handlungszusammenhänge vorgibt und das Repertoire und die Sequenz der konstitutiven Elemente dieses kommunikativen Vorgangs festlegt (Ort und Verlauf einer Vorlesung, Begrüßung, Einstieg ins Thema, Präsentation eines Themas, Beendigungssequenz, Abwesenheit von Hörersignalen wie "mhm" oder "genau " etc.), sondern auch den an der Kommunikation Beteiligten verhältnismäßig kon-

1 Es bestehen hier - wie auch in zahlreichen anderen Aspekten - Ähnlichkeiten zum Konzept der »sprachlichen Handlungsmuster« von Ehlich/Rehbein, d.h. zu jenen »Formen von standardisierten Handlungsmöglichkeiten, die im konkreten Handeln aktualisiert und realisiert werden« (1979: 250).

2 Vgl. in diesem Zusammenhang auch Fix (1998: 17) zum »Textsortenwissen« als »Teil unserer im sozialen Handeln erworbenen Kompetenz «. 
stante Beziehungsmuster (Professor/in vs. Student/in) zuordnet.

Gattungen bezeichnen also kommunikative Vorgänge, in denen bestimmte sprachlich-kommunikative Elemente zusammengefügt und in ihren Anwendungsmöglichkeiten vorgezeichnet sind. In gewissen Situationen (z. B. um den Beginn eines gemeinsamen Essens und zugleich die religiöse Zugehörigkeit zu markieren) liegt es aufgrund des vorhandenen kommunikativen Problems für die Interagierenden nahe, eine spezifische Gattung (ein Tischgebet) zu verwenden. In anderen Situationen (beispielsweise wenn Sprecher/innen die Handlung ihres Gegenüber kritisieren möchten) bleibt ihnen die Wahl zwischen mehreren möglichen Gattungen und kleinen Formen wie z. B. Vorwürfen, Frotzeleien, sich mokieren, Beschwerden etc. (vgl. Christmann 1995, Günthner 2000). Die Wahl hängt vom größeren sozialen Kontext, von der spezifischen Sprechsituation, den betreffenden Teilnehmer/innen, den konkreten Intentionen der Sprecher/innen, dem Habitus etc. ab. Gattungen sind also nicht etwa als komplexe, von der Interaktion losgelöste Sprachstrukturen, sondern als im Sprechen erzeugte interaktive Handlungsmuster zu betrachten. ${ }^{1}$ Orientieren sich die Interagierenden an einer Gattung, so können sie sich eng an die Gattungsregeln halten, diese etwas abändern oder gar mit ihnen »spielen«. D. h. sie können mehr oder weniger prototypische Formen der betreffenden Gattung aktualisieren. ${ }^{2}$

Gattungen fungieren also als Orientierungsrahmen, auf die sich Interagierende sowohl bei der Produktion kommunikativer Handlungen als auch bei der Rezeption beziehen: Wenn beispielsweise eine Sprecherin ihre Äußerung mit »Kennst du den schon? Kommt Fritzchen nach Hause... « beginnt, so erwarten wir in der Regel einen Witz. Hören wir »Hast du schon gehört: die Frau Müller ...", so erzeugt auch hier die Sprecherin bestimmte Gattungserwartungen, nämlich in Richtung Klatsch und Tratsch. Diese Gattungserwartungen beziehen sich einerseits auf die Form (d.h. wir erwarten im Falle von Klatsch eine Ereignisrekonstruktion über eine uns bekannte, abwesende Person), andererseits aber auch auf den Inhalt (im Falle von Klatsch erwarten wir eine Neuigkeit über das (Privat)-Leben des Klatschobjektes; zur kommunikativen Gattung des Klatsches siehe Bergmann 1987). Wir haben also eine bestimmte Vorstellung vom »Redeganzen « (Bachtin 1979/86: 79). Das Wissen, daß kommunikative Vorgänge mit bestimmten Funktionen in typischen Situationszusammenhängen auf bestimmte Weise verlaufen, steuert also nicht nur das kommunikative Handeln selbst, sondern auch die Deutung dieses Handelns; d.h. die Interagierenden orientieren sich nicht nur bei der Produktion ihrer Äußerungen, sondern auch bei der Interpretation der Äußerungen des Gegenüber an ihrem Gattungswissen (Günthner / Knoblauch 1994, 1995).

Die Erforschung mündlicher (und auch schriftlicher) Gattungen ist für die Analyse von Kommunikationssituationen u. a. deshalb so zentral, weil das Wissen um die betreffende Gattung eine Äuße-

1 Vgl. hierzu Günthner/Knoblauch (1994), (1995); siehe auch Parallelen zu Ehlich/ Rehbeins (1979) Konzept der »sprachlichen Handlungsmuster«.

2 Das vorliegende Gattungskonzept, das auf die Analyse interaktiver Handlungsmuster abzielt, geht über eine rein formale Betrachtung sprachlicher Textmerkmale hinaus und wendet sich gegen eine statische Gattungsbetrachtung, die stilistische Homogenität und Nichtüberlappung propagiert (Günthner 1995). 
rung häufig erst interpretierbar macht. $\mathrm{Ob}$ wir einen Text als Fiktion, Parodie, Gebet oder Klatsch lesen bzw. hören, ist eine Gattungsfrage, die wichtige Konsequenzen für die Interpretation hat (Hanks 1987: 670).

Ferner sind Gattungen weitgehend „soziokulturell abgeleitet «, d.h. sie werden nicht von einzelnen Interagierenden ständig neu konstituiert, sondern vom gesellschaftlichen Wissensvorrat bereitgestellt (Luckmann 1988). Folglich ist es naheliegend, daß unterschiedliche Kulturen bestimmte kommunikative Aufgaben unterschiedlich lösen. D. h. das Repertoire der Gattungen (wie auch der schriftlichen Textsorten) variiert von Kultur zu Kultur. ${ }^{1}$

Mitglieder einer Kultur verfügen in der Regel über das für sie notwendige Gattungswissen: Sie wissen beispielsweise, wie man klatscht, sie erkennen Klatsch, und sie wissen auch, in welchen Situationen und wem gegenüber welche Art von Klatsch angebracht bzw. zu vermeiden ist. In interkulturellen Situationen treffen nun Interagierende aufeinander, die über unterschiedliche »Ausgangs«-Repertoires an kommunikativen Formen und Gattungen verfügen. So kann in einer Kultur ein bestimmtes kommunikatives Problem (z. B. die Auswahl eines Kandidaten für einen Arbeitsplatz) gattungsmäßig verfestigt sein (in Form von Bewerbungsgesprächen), während in einer anderen Kultur diese Gattung nicht existiert, wie beispielsweise in der VR China und Vietnam, wo es bis vor kurzem keinen freien Arbeitsmarkt gab. Besonders trügerisch für interkulturelle Begegnungen sind jedoch kulturell unterschiedliche Realisierungsformen scheinbar glei- cher Gattungen, wie kulturelle Unterschiede bei wissenschaftlichen Vorträgen (vgl. Kotthoffs Arbeit zu stilistischen Unterschieden in russischen und deutschen wissenschaftlichen Vorträgen 1999), bei universitären Prüfungsgesprächen, bei Geschäftsverhandlungen (vgl. Li 1999) etc. $^{2}$

Die Realisierung bestimmter Gattungen und damit verbunden die Gattungsregeln - betreffen, wie bereits die Textlinguistik in Zusammenhang mit Textsorten verdeutlicht hat, unterschiedliche Ebenen. In der Textlinguistik wurde traditionellerweise zwischen textinternen und textexternen Elementen der Konstitution von Textsorten unterschieden. Im folgenden sollen jedoch (in Anlehnung an Luckmann 1988 und Günthner/Knoblauch 1994, 1995) folgende drei Ebenen unterschieden werden, die für die Aktualisierung einer Gattung von Relevanz sind:

1. die Ebene der Binnenstruktur;

2. die interaktive Realisierungsebene;

3. die Ebene der Außenstruktur.

1. Die Binnenstruktur einer Gattung besteht aus jenen »textinternen «, verbalen und nonverbalen Elementen, die für die betreffende Gattung konstitutiv sind. Hierzu zählen prosodische Phänomene (Intonation, Lautstärke, Sprechgeschwindigkeit, Pausen, Rhythmus, Akzentuierung), Aspekte der Stimmqualität, gestisch-mimische Elemente, phonologische Variationen, lexiko-semantische Phänomene und morpho-syntaktische Elemente. Auch die Wahl einer spezifischen sprachlichen Varietät, stilistische und rhetorische Figuren sind auf dieser Ebene angesiedelt. Ferner können aber auch bereits verfestigte »Klein-«

1 Günthner/Luckmann im Druck 2000; siehe hierzu u. a. Fix 1998, die Textsorten als »kulturell geprägte Phänomene« beschreibt.

2 Vgl. auch die kontrastiven Arbeiten von Galtung (1985) und Clyne (1987), (1991) in Zusammenhang mit wissenschaftlichen Textsorten; hierzu auch Ehlich (1998). 
und »Kleinstformen « - wie verbale Stereotype, idiomatische Redewendungen, Gemeinplätze, Sprichwörter, formularische Ausdrücke, historisch tradierte Formeln und Rätsel - zum Repertoire der Binnenstruktur einer Gattung zählen. Auch inhaltliche Verfestigungen, die sowohl Themen als auch Motive, Figuren oder Schauplätze betreffen, gehören zur Binnenstruktur kommunikativer Gattungen. Das Medium (mündlich bzw. schriftlich, face-to-face bzw. medial vermittelt) sowie die Interaktionsmodalität bilden weitere konstitutive Merkmale einer Gattung. Beispielsweise zeichnen sich »Frotzeleien « durch eine Ambivalenz der Interaktionsmodalität von Spaß und Ernst aus (Günthner 2000). Hierbei wird einerseits eine Verfehlung bzw. ein inadäquates Verhalten des Frotzelobjekts vorgeführt, wodurch die Frotzeläußerung einen mehr oder weniger stark gesichtsbedrohenden und irritierenden Charakter erhält. Andererseits erfährt die Provokation jedoch eine Abschwächung und Milderung aufgrund der Spiel- und Spaßmodalität; d.h. die Stärke der Provokation, Unhöflichkeit und Kritik wird gebrochen durch die spielerisch-spaßhafte Interaktionsmodalität.

2. Während also die Binnenstruktur die »textinternen « Elemente von Gattungen umfaßt, gehören jene Phänomene, die den interaktiven Kontext des dialogischen Austauschs zwischen mehreren Interagierenden und die Sequentialität von Äußerungen betreffen, zur interaktiven Realisierungsebene kommunikativer Gattungen (Günthner/Knoblauch 1994). Gerade die Arbeiten der Konversationsanalyse, die die sequentielle Organisation sprachlicher Handlungen, das System des Redewechsels, Paarsequenzen, Prä-, Post- und Einschubsequenzen und Präferenzstrukturen beschrieben haben, sind für diese strukturelle Ebene von besonderer Bedeutung. Auch Präferenzstrukturen sind eng mit der betreffenden Gattung verbunden. Pomerantz' (1984) Arbeit zu konversationellen Bewertungen verdeutlicht, daß in smalltalk-Situationen eine Präferenz für $\mathrm{Zu}$ stimmung herrscht und Nichtübereinstimmungen in ein dispräferiertes Format gekleidet werden. In Argumentationen dagegen ändern sich die Präferenzstrukturen und nicht-übereinstimmende Äußerungen, in denen die Polarität zur vorherigen Aussage geradezu hervorgehoben und fokussiert wird, sind hier präferiert (Kotthoff 1993). Zur interaktiven Realisierungsebene gehört auch das Äußerungsformat einer Gattung, das anzeigt, in welcher Beziehung die Sprechenden zu dem kommunizierten Sachverhalt bzw. den zitierten Figuren oder Charakteren stehen. Durch das Äußerungsformat signalisieren die Sprecher/ innen, ob sie andere Personen zitieren, als Sprachrohr fungieren (wie beispielsweise bei Nachrichtensprecher/innen im Fernsehen) oder aber eigene Meinungen, Erfahrungen etc. ausdrücken. Auch der Teilnehmerstatus, der das Verhältnis der Kommunizierenden zueinander und $\mathrm{zu}$ ihren Äußerungen betrifft, ist auf dieser Ebene angesiedelt. Dieser zeigt an, welche Gesprächsrollen die Interagierenden während der Durchführung einer Gattung einnehmen (z. B. als Prüfer und Prüfling; als Ärztin und Patient). 3. Die Außenstruktur kommunikativer Gattungen bezieht sich auf den Zusammenhang von Gattungen und sozialen Milieus, ethnischen und kulturellen Gruppierungen, Geschlechterkonstellationen, Institutionen etc. Gattungen sind häufig nicht nur bestimmten sozialen Milieus zugeordnet, sondern soziale Milieus, wie beispielsweise Familien, Frauen- und Ökogruppen oder Studentencliquen, zeichnen sich durch typische, immer wiederkehrende soziale 
Veranstaltungen aus, in denen typische kommunikative Gattungen anzutreffen sind und wiederum andere fehlen (Schwitalla 1995, Keim 1997, Christmann 1999). Auch kulturelle Gruppen oder soziale Milieus (weiße und schwarze US-Amerikaner/innen, Briten und Inder/innen in Großbritannien, deutsche und chinesische Studierende) weisen Unterschiede im Gebrauch bestimmter kommunikativer Gattungen (wie z. B. Argumentationen, Jobinterviews und Seminarvorträge) auf. Diese Unterschiede betreffen nicht nur Aspekte der Binnenstruktur und der situativen Realisierungsebene (Organisation des Arguments, prosodische Merkmale zur Signalisierung bekannter und neuer Informationen, Rezipientenreaktionen etc.), sondern auch die kommunikative Funktion dieser Gattungen (vgl. hierzu auch die Arbeiten von Kallmeyer (1994) und Schwitalla (1994)). Aber auch geschlechtsbedingte Unterschiede zeichnen sich anhand kommunikativer Gattungen ab (Gal 1989, Günthner/Kotthoff 1991, Baron 1998). Im kaukasischen Georgien bildet beispielsweise die Gattung der Trinksprüche eine wichtige ritualisierte Handlung und ein zentrales Mittel zur interaktiven Konstruktion von »Männlichkeit«. Trinksprüche können sich in bestimmten Kontexten sogar zu einem Wettstreit unter den am Tisch anwesenden Männern entwickeln, wobei jene Georgier, die die Gattung nicht beherrschen und denen es an rhetorischen Fertigkeiten mangelt, als »unmännlich « klassifiziert werden (Kotthoff 1991). Während in informellen Situationen gelegentlich auch Frauen die Rolle des Trinkspruchmeisters übernehmen können, ist dieser in formellen Situatio- nen stets ein Mann. Kommunikative Gattungen haben auch bei der Konstruktion spezifischer institutioneller Kontexte eine zentrale Rolle inne: Beispielsweise zeichnet sich der universitär-wissenschaftliche Bereich durch die Verwendung bestimmter akademischer Gattungen (Seminardiskussionen, Prüfungsgespräche, Vorlesungen, Referate, Klausuren, Abstracts, wissenschaftliche Aufsätze, Rezensionen etc.) aus, die wie Swales (1990) verdeutlicht - kulturell unterschiedlich organisiert sein können. ${ }^{1}$

Die Außenstruktur vermittelt somit zwischen kommunikativen Aktivitäten und der Sozialstruktur einer Gesellschaft. Die soziale Position, die kulturelle $\mathrm{Zu}$ gehörigkeit, das soziale Milieu etc. haben einen erheblichen Einfluß auf das Repertoire kommunikativer Gattungen einzelner Mitglieder und dieses wiederum regelt den Zugang zu gesellschaftlichen Positionen, Milieus etc. Kenntnisse kommunikativer Gattungen sind nicht nur Teil der »kommunikativen Kompetenz«, sondern auch Teil des »kommunikativen Kapitals« (Bourdieu 1990), und entscheiden häufig - wie zahlreiche Arbeiten im Bereich der interkulturellen Kommunikation verdeutlichen - über Erfolg oder Mißerfolg in institutionellen Kontexten (Gumperz 1982).

Die drei hier aufgezeigten Ebenen (die Ebene der Binnenstruktur, die interaktive Realisierungsebene und die der Außenstruktur) konstituieren das Gesamtmuster einer kommunikativen Gattung. Interagierende können sich relativ eng an die Gattungsvorgaben anlehnen und damit Gattungen in ihrer prototypischen Form aktualisieren; sie können aber auch

1 Vgl. auch Ehlich (1998: 863) zu kulturspezifischen Wissenschaftstraditionen und Anforderungen an eine »Wissenschaftssprach-Komparatistik«. 
von den Vorgaben abweichen bzw. mit ihnen »spielen « (hierzu detailliert Günthner 2000).

\section{Aspekte der Aktualisierung kommu- nikativer Gattungen in der Interkultu- rellen Kommunikation}

Als kulturelle und historische Produkte sind Gattungen offen für kulturelle Variation und historischen Wandel (Luckmann 1988). In interkulturellen Kommunikationssituationen treffen folglich Interagierende mit einem unterschiedlichen Repertoire an kommunikativen Gattungen aufeinander (Günthner 1993, Günthner/ Luckmann 2000). Im folgenden sollen exemplarisch einige Konsequenzen kulturell unterschiedlicher Gattungstraditionen für die interkulturelle Kommunikation beleuchtet werden.

\subsection{Nicht-Verfügen über die betreffende Gattung}

Gattungstraditionen verschiedener kultureller Gruppen können sich dadurch unterscheiden, daß in einer Gruppe ein bestimmtes kommunikatives Problem gattungsmäßig verfestigt ist, d.h. daß für eine immer wiederkehrende kommunikative Aufgabe eine bestimmte konventionalisierte Form zur Lösung dieser Aufgabe zur Verfügung steht, während eine andere Kulturgemeinschaft keine feste Gattung für das betreffende Problem hat.

So veranschaulicht Auers (1998) Analyse von Bewerbungsgesprächen mit ost- und westdeutschen Teilnehmenden, daß die ostdeutschen Bewerber/innen mit dieser institutionellen Gattung Schwierigkeiten haben, da in der ehemaligen DDR Bewerbungsgespräche nicht Teil des kommunikativen Haushalts waren. Durch die Wiedervereinigung wurden nun die Bewerber/innen aus den neuen Bundesländern mit einer
Gattung konfrontiert, die bislang nicht zu ihrem kommunikativen Repertoire gehörte. Sie »lösten « - wie Auers (1998) Analysen zeigen - dieses Problem, indem sie Strategien, die dem offiziellen Sprachgebrauch der ehemaligen DDR entsprachen, anwendeten und diese Strategien wiederum mit Techniken mischten, von denen sie annahmen, daß sie in dieser »westlichen « Gattung verlangt wurden. Folglich kommt es in diesen Gesprächen zu einer Spannung zwischen westlichen und östlichen Diskursstrategien: Während Bewerbungsgespräche im Westen eine explizite Demonstration von Wissen und Erfahrung verlangen, erwartet der ostdeutsche Diskurs die Demonstration von Bescheidenheit; die westliche Gattung verlangt aktives Engagement der Bewerber, im »Oststil« wird dagegen das Agent-Subjekt vermieden; während die westliche Gattung auf einer Ideologie der »scheinbaren Gleichheit « basiert, die die darunterliegenden Machtverhältnisse verdekken soll, exponiert der »Oststil« diese Machtstrukturen durch einen hohen Formalitätsgrad und die Signalisierung von Unterwürfigkeit; während der westliche Stil Direktheit verlangt, erfordert der östliche Vagheit und Indirektheit; Bewerbungsgespräche im Westen verlangen die Fähigkeit, die Interaktionsmodalität spontan zu wechseln und in eine informelle und spaßhafte Interaktionsphase überzutreten; dagegen zeichnet sich der östliche Bewerbungsdiskurs durch Steifheit und Unflexibilität aus. Die Bewerber/innen aus den neuen Bundesländern konstruieren folglich ihre kulturelle Zugehörigkeit durch bestimmte Diskursverfahren, die der westlichen Gattung des Bewerbungsgesprächs nicht entsprechen. All dies hat wie Auer (1998) zeigt - Konsequenzen für den Verlauf und das Resultat der 
Bewerbungsgespräche (vgl. auch Kern 2000).

Aber auch innerhalb des universitären Bereichs zeigen sich erhebliche kulturelle Unterschiede im Repertoire kommunikativer Gattungen. ${ }^{1}$ Beispielsweise ist bei uns die universitäre Sprechstunde eine feste Institution. Studierende erwerben relativ rasch Erfahrungen im Umgang mit der kommunikativen Gattung der Sprechstundengespräche. Diese umfaßt Wissen darüber, daß ein Sprechstundengespräch im Büro einer Professorin/eines Dozenten stattfindet, $\mathrm{da}$ es zeitlich festgelegt ist (d.h. an einem bestimmten Wochentag und $\mathrm{zu}$ einer bestimmten Stunde stattfindet) und oft zeitlich stark begrenzt ist und $\mathrm{da}$ es feste kommunikative Rollen beinhaltet (Professor vs. Student). Ferner gehört Wissen dazu, wie man ein solches Gespräch beginnt, welche Fragen und Probleme man dort bespricht und welche nicht (z. B. daß man in der Regel die Professorin nicht nach deren Familiensituation befragt oder danach, wie es ihr gesundheitlich geht, etc.) und wie ein solches Gespräch beendet wird. In anderen Kulturgemeinschaften (wie beispielsweise in China und Vietnam) existiert die Gattung der Sprechstundengespräche nicht. Dies kann dazu führen, daß Studierende aus diesen kulturellen Kontexten zunächst Probleme mit der Institution "Sprechstunde « und der kommunikativen Gattung des Sprech- stundengesprächs an deutschen Hochschulen haben. Die folgenden Ausschnitte, die Gesprächen und Interviews entstammen, die ich in den Jahren 1990 bis 1996 mit chinesischen Studierenden an deutschen Hochschulen durchgeführt und aufgezeichnet habe, illustrieren diese Problematik.

So berichtet $\mathrm{Fan}^{2}$, eine chinesische Germanistin:

»Diese Sprechstunden, die gibt es bei uns in China nicht. In China besuchen die Studenten den Prof. zu Hause. Das bedeutet: man hat was, man will was von dem Lehrer. Normalerweise. Als ich dann nach Deutschland kam, wußte ich wirklich am Anfang gar nicht, was man in diesen Sprechstunden macht. Ich kannte die Form Sprechstunde überhaupt nicht. Und da hab ich dann andere Chinesen gefragt, was man denn in den Sprechstunden eben reden sollte. Und da haben die gesagt: übers Studium. Man sollte denen erzählen, was man alles gemacht hat. Man geht mit einem Ziel hin. Und man muß eben das Ziel erreichen, wenn man rauskommt.«

Han (ein chinesischer Doktorand) erzählt:

"Ja, am Anfang ist man sehr unsicher. Was ist so eine Sprechstunde? Was macht man da? Da habe ich einen chinesischen Kommilitonen gefragt. Der hat gesagt: $>$ Ja du sollst dem Professor sagen, du hast alles gelernt und kannst alles und willst gleich mit der Diss. anfangen $<$. Und das habe ich dann gemacht. Aber das war wohl nicht so ganz gut. Bei uns besucht man den Professor zu

1 Vgl. Swales (1990), der verschiedene (schriftliche) wissenschaftliche Textgattungen (wissenschaftliche Artikel, Abstracts, Projektanträge, Thesen, Dissertationen und Anfragen um Sonderdrucke) analysiert und zeigt, wie diese im anglo-amerikanischen Kontext prototypisch realisiert werden. Darüber hinaus thematisiert er kulturelle Unterschiede im Umgang mit diesen Textgattungen und verweist auf Schwierigkeiten von non-native speakers, die über andere Gattungskonventionen verfügen und folglich im amerikanischen System Schwierigkeiten haben. Siehe auch Ehlich (1998) $\mathrm{zu}$ kulturspezifischen Wissenschaftstraditionen und Wissenschaftssprachen sowie Kotthoff (1999) zu kulturspezifischen Vortragsstilen bei wissenschaftlichen Konferenzen.

2 Die verwendeten Namen stellen Pseudonyme dar. 
Hause und bringt Geschenke mit. Auch beim Parteisekretär ist das so.«

Li, eine chinesische Studentin, die an einer deutschen Hochschule studiert, berichtet von ihrer ersten Sprechstundenerfahrung in Deutschland:

»Ich bin dann zu dem Professor gegangen und hab' ihm eine Lackvase mitgebracht, und hab' gesagt: >Ich möchte Ihnen eine chinesische Vase schenken<. Doch er hat die Vase angeguckt und hat gesagt, >Frau Li, das ist sehr nett von Ihnen, aber ich kann die Vase nicht annehmen die Vase dann wieder zurücknehmen. Und da war ich schockiert. Und ich hab gedacht: $\mathrm{Du}$ bist aber blöd. Gleich beim ersten Mal hast du die Gelegenheit versaut. hihihi. [...] Bei uns legt man ziemlich viel Wert darauf, daß der Professor das Geschenk annimmt. Ich hab' erst viel später kapiert, daß man das hier nicht macht. [...] In China denkt man so: Man soll das Geschenk zuerst geben und dann über die Sache, die man will, reden. Damit das besser läuft. Nicht umgekehrt.«

Diese Gesprächsausschnitte verdeutlichen nicht nur Unsicherheiten chinesischer Studierender im Umgang mit einer ihnen bislang unvertrauten kommunikativen Gattung, sondern sie verweisen auch darauf, daß bestimmte kulturelle Erwartungen, die diese Studierenden an die fremde Situation haben - wie daß man dem Professor Geschenke macht, damit das Gespräch angenehmer verläuft - unter Umständen enttäuscht werden.

\subsection{Scheinbar gleiche Gattungen wer- den unterschiedlich realisiert}

Ein weiteres Problem für die interkulturelle Kommunikation tritt dann auf, wenn scheinbar gleiche Gattungen kulturell unterschiedlich realisiert werden.

So zeigen Lis Analysen chinesisch-holländischer/finnischer Geschäftsverhandlungen (1999) kulturelle Differenzen in Bezug auf die kommunikative Gattung der »business negotiations«.
Die Unterschiede betreffen einerseits kulturspezifische Erwartungen an Geschäftsverhandlungen: Während chinesische Teilnehmer/innen bei den Verhandlungen großen Wert auf die Etablierung zwischenmenschlicher Beziehungen legen, steht bei den holländischen und finnischen Partner/innen der Geschäftsabschluß sehr viel stärker im Zentrum ihres kommunikativen Vorgehens. Zum anderen zeigen sich Unterschiede in der Diskursorganisation: Die chinesischen Geschäftspartner tendieren dazu, zunächst Hintergrundinformationen zu präsentieren, bevor die zentrale Information kommt. Oft liefern sie nur die Hintergrundinformationen und erwarten, daß die Geschäftspartner/innen selbst die notwendigen Schlußfolgerungen ziehen. Diese kulturell divergierenden Vorstellungen bezüglich der Gattung »Geschäftsverhandlungen« können dazu führen, daß in interkulturellen Verhandlungsgesprächen Unsicherheiten, Frustrationen und die Erfahrung von »Fremdheit « aufkommen.

Auch Kotthoffs (1999) Untersuchung zu deutsch-russischen Unterschieden bei (geistes-)wissenschaftlichen Vorträgen verweist auf kulturspezifische Differenzen in der Handhabung scheinbar gleicher Gattungen. Während die deutschen Wissenschaftler/innen den Zuhörenden eine Orientierung zum thematischen Aufbau des Vortrags und zur Spezifik der Fragestellung liefern, fehlt in den russischen Vorträgen in der Regel eine solche Orientierungshilfe. Ferner sind die deutschen Vorträge thematisch meist stark fokussiert, während der thematische Skopus der russischen Vorträge sehr weit reicht (und in seiner thematischen Weite eher einem Festvortrag bei uns entspricht). Während bei den deutschen Vorträgen Unterthemen meist explizit genannt werden, ist dies bei den russischen nicht der Fall. Auch ist bei 
den russischen Vorträgen nur schwer auszumachen, was eigene Thesen und Gedanken sind und was von anderen Forschenden stammt. $^{1}$

\subsection{Unterschiedliche Funktionen und Bewertungen scheinbar gleicher Gat- tungen}

Ein weiterer für die interkulturelle Kommunikation relevanter Aspekt der Gattungsforschung betrifft kulturell unterschiedliche Funktionen und Bewertungen scheinbar gleicher kommunikativer Gattungen.

Betrachten wir hierzu kulturelle Unterschiede in der Verwendung der kleinen Form bzw. primären Gattung (Bachtin 1979/86) der Sprichwörter. Während Sprichwörter ${ }^{2}$ im deutschen Kontext als Träger sozial verbindlicher Normen eine »Volksmoral im weiteren Sinne zum Ausdruck bringen " (Bausinger 1968), als Weisheit »des kleinen Mannes«, und Stilwörterbücher geradezu vor dem Gebrauch von Routineformeln und Sprichwörtern als Zeichen »schlechten Geschmacks « warnen, wird im chinesischen Sprachgebrauch die Fähigkeit, in der richtigen Situation die adäquate For- mel zu finden, geradezu honoriert (Günthner 1993). Sprichwörter gelten als Zeichen hoher Bildung: die Sprecher/innen verdeutlichen durch die Verwendung dieser - im chinesischen Kontext - prestigereichen kleinen Form ihre enge Verbundenheit mit kulturellen Normen und Weisheitstraditionen. Arbeiten zur chinesischen Rhetorik, Studien zur Umgangssprache sowie Chinesischlehrbücher für Ausländer betonen stets die lebendige Sprichwortkultur im Chinesischen und die Beliebtheit des Sprichwortgebrauchs in mündlichen und schriftlichen Texten.

In interkulturellen Kommunikationssituationen zwischen Deutschen und Chinesen/innen verwenden chinesische Sprechende häufig (chinesische und deutsche) Sprichwörter zur Stütze von Meinungskundgaben, Bewertungen, Urteilen sowie anstelle einer persönlichen Meinungskundgabe. Dies soll anhand eines Beispiels illustriert werden.

Der folgende Transkriptausschnitt ist einem Gespräch zwischen der Chinesin Wang und der Deutschen Lisa entnommen. Lisa thematisiert, daß sie oft bei Chinesen den Eindruck hat, sie wollen

1 Vgl. in diesem Zusammenhang auch Liangs Analysen der Textsorte Rezensionen im Chinesischen und Deutschen (1991): Chinesische Rezensionen zeichnen sich vor allem dadurch aus, daß sie versuchen, das Positive zu entdecken und hervorzuheben, und folglich die Würdigung der Publikation im Fokus einer Rezension steht. Bei den deutschen Rezensionen - so Liang (1991) - steht dagegen die kritische Auseinandersetzung im Vordergrund: Häufig werden Details kritisiert und Aspekte der Arbeit explizit negativ bewertet. »Versöhnende bzw. tröstende Worte « finden sich bei den deutschen Rezensionen oft erst gegen Textende, und selbst dann werden diese »durch einen Hinweis auf die Negativa wieder relativiert«. Siehe auch Gnutzmanns Arbeit zu Unterschieden zwischen deutschen und englischen rabstracts< im Fach Maschinenbau (1991) sowie Eßers Studie zu geisteswissenschaftlichen Arbeiten deutscher und mexikanischer Studierender (1997) und Günthners Arbeit zu deutsch-chinesischen Unterschieden im Abfassen von Seminar- und Magisterarbeiten (1988).

2 Ich verwende hier den Sprichwort-Begriff im engeren Sinne (hierzu Gülich 1978, Lüger 1999). Sprichwörter sind satzwertige, reproduzierbare Phraseologismen, die sich durch figurative, metaphorische Sprache auszeichnen, eine ganzheitliche (idiomatische) Bedeutung haben, Lebensweisheiten und -erfahrungen zum Ausdruck bringen und volksläufig sind. Sie sind folglich von nicht-bildhaften Gemeinplätzen und (nichtsatzwertigen) Redewendungen zu unterscheiden. 
einem ihre Meinung nicht sagen bzw. »haben keine eigene Meinung zu vielen Themen«. Daraufhin kommt eine kurze
Sequenz darüber, wie direkt man in China und in der Bundesrepublik seine Meinung äußern kann:

\begin{tabular}{||l|l||}
\hline \multicolumn{2}{|l||}{ WANG 22 } \\
\hline 12 Lisa: & also das is (-) ja dann schon so \\
\hline 13 & auch bei euch in SCHINA \\
\hline 15 & daß man erwartet daß eh jemand ne eigene MEINUNG HAT, \\
\hline 16 & oder $?$ \\
\hline 17 Wang: & schon so. (-) ja. ehm jeder- jemand läßt sich IMMER eh ja, \\
\hline 18 & =wie=sagt=man= ja eh sehr leicht ÜBERREDEN \\
\hline 19 & er hat keinen eigenen Mein- MEINUNG ja, \\
\hline 20 Lisa: & mhm \\
\hline 21 Wang: & man fragt was für eine MENSCH ist DAS? \\
\hline 21 & solche Menschen wir sagen ehm, \\
\hline 22 & sind ja mo- MORALISCH ${ }^{\circ}$ nich so ganz gut ${ }^{\circ}$ \\
\hline 23 & solche MENSCHEN ja. man sagt bei uns, \\
\hline 24 & $(0.5)$ \\
\hline 25 & wir haben so eine Sprich' eh Spruchwort das heißt (0.5) \\
\hline 26 & QIANGSHANG YIGEN CAO \\
\hline 27 & FEN ZUI LIANGBIAN DAO. \\
\hline 28 & das bedeutet so ja. diese Menschen \\
\hline 29 & richten sich nur nach MACHT. ja? \\
\hline 30 Lisa: & nach der MACHT? \\
\hline 31 Wang: $:$ & ja. ja. nach der MACHT und so. \\
\hline 32 Lisa: & hm? \\
\hline 33 Wang: $:$ & also nach MÄCHTIGE LEUTE. \\
\hline 34 & das heißt sie haben keine eigenen Charakter oder so \\
\hline 35 & so eigene Meinung. \\
\hline 36 Lisa: & ahja. \\
\hline 37 Wang: $:$ & deshalb bei uns diese Menschen ja sie haben so eine \\
\hline 38 & Spitzname QIANGTOU CAO. \\
\hline
\end{tabular}


Das moralische Urteil »solche Menschen wir sagen ehm sind ja mo- MORALISCH nich so ganz gut « (21-22) wird durch das Zitieren eines chinesischen Sprichwortes untermauert (25-26). Die chinesische Redewendung (QIANGSHANG YIGEN CAO FEN ZUI LIANGBIAN DAO - »ein Grashalm auf der Mauer legt sich auf jede Seite, die dem Wind gefällt «) hebt sich vom umliegenden Kontext als »fremde Stimme«ab.

Sprichwörter werden von chinesischen Interagierenden jedoch nicht nur in informellen Kommunikationssituationen zitiert, sondern auch im wissenschaftlichakademischen Kontext, in Vorträgen, Magisterarbeiten und Aufsätzen (Jia 1999). In interkulturellen Situationen übertragen chinesisch Sprechende und Autoren/innen häufig eigene Konventionen der situativen Verwendung dieser kleinen Formen ins Deutsche: Auf Deutsch verfaßte wissenschaftliche Vorträge und schriftliche Texte beginnen und enden nicht selten mit einem deutschen Sprichwort (wie »Besser den Spatz in der Hand als die Taube auf dem Dach «, » Der Mensch denkt - Gott lenkt«, oder »Mit Speck fängt man Mäuse«). Dieser Transfer der eigenen Gattungskonventionen sowie deren Bewertung in die Fremdsprache (hier: ins Deutsche) wird auch dadurch verstärkt, daß an zahlreichen chinesischen Universitäten das Lernen und Prüfen von Sprichwörtern in der Fremdsprache Teil der Studienanforderungen darstellen (Günthner 1993).

\subsection{Hyperkorrekturphänomene bei der Aktualisierung kommunikativer Gat- tungen}

Ein weiterer in Zusammenhang mit der Analyse kommunikativer Gattungen zu berücksichtigender Aspekt ist, daß Teilnehmer/innen interkultureller Kommunikationssituationen in der Regel keineswegs immer ohne Vorwissen über die »andere Kultur«, die »fremdkulturellen
Verhaltensweisen und Eigenschaften", »andere sprachliche Etikettenregeln und Gesprächsnormen « die Interaktion betreten. Vielmehr haben sie häufig bestimmte Erwartungen, da sie bereits über eigene fremdkulturelle Erfahrungen verfügen oder aber via Dritte über »andersartiges Verhalten " informiert wurden. Sie wissen, daß ihr Gegenüber sich in bestimmten kommunikativen Situationen "anders « verhält. Dieses Wissen fließt in die Interaktion mit ein und führt nicht selten zu bestimmten »interaktiven Hyperkorrekturphänomenen «bzw. zur »Adaption an die Erwartung fremdkulturellen Verhaltens « (Günthner 1993, 1999). Das heißt Interagierende überformen eigene Verhaltensweisen, indem sie sich an vermeintlich fremde Konventionen adaptieren. So wird beispielsweise chinesischen Studierenden in China nahegelegt, im Umgang mit Deutschen »direkt « zu sein, da die Deutschen angeblich »Direktheit « erwarten. Doch wird dabei weder genauer erläutert, worin die »Direktheit « besteht, noch auf welche kommunikativen Situationen (Gattungen, Teilnehmerkreis etc.) sie sich bezieht. Die Konsequenz ist, daß zahlreiche Chinesen/innen, die versuchen, diesen Erwartungen $\mathrm{zu}$ entsprechen, den Boden eigener Konventionen verlassen, ohne jedoch den für die jeweilige kommunikative Gattung adäquaten »Direktheitsstil « im Deutschen zu erreichen. Stattdessen entsteht dann ein Verhalten, das in den Augen ihrer deutschen Kommunikationspartner/innen »unverfroren « wirkt. Eine Analyse von Briefen, die chinesische Studierende und Wissenschaftler an deutsche Professoren schrieben, um sich über Möglichkeiten eines Forschungsplatzes oder einer Doktorandenstelle zu erkundigen, verdeutlicht solche »Adaptionsstrategien «. Diese Briefe bestanden nicht selten aus einer einleitenden Entschuldigungsformel für »die Störung « plus fol- 
gender Aneinanderreihung von Forderungen und Wünschen an den deutschen Wissenschaftler. Um ein Beispiel zu geben (bei dem Namen und Fachrichtung geändert wurden):

Sehr geehrter Herr Prof. Dr. Schmidt! Verzeihen Sie, daß ich Sie mit meinem Anliegen störe! Mein Name ist Liu Xiaobing. Ich arbeite als Dozent für Physik an der Hochschule in Nanjing. Ich möchte gerne bei Ihnen arbeiten. Ich brauche deshalb einen Laborplatz bei Ihnen. Können Sie ihn mir zur Verfügung stellen. Leider dauert mein Stipendium von der chinesischen Regierung nur ein Jahr. Doch ich möchte meine Doktorarbeit bei Ihnen schreiben. Bitte seien Sie so höflich und besorgen Sie mir ein Stipendium für die Doktorarbeit. Auch wichtige Forschungsliteratur ist in China nur schwierig zu bekommen. Deshalb brauche ich neuere Literatur von Ihnen. Schicken Sie die Bücher an meine Adresse in Nanjing.

Entschuldigen Sie meine Belästigung.

Die Frage, ob die betreffenden Verfasser ihre Briefe auf Chinesisch ähnlich abfassen würden, wurde von ihnen stets verneint. Man habe gehört, im Westen könne man sehr direkt sein, deshalb habe man so offen geschrieben.

Dieses Beispiel veranschaulicht die Komplexität des Aufeinandertreffens verschiedener Gattungstraditionen in der interkulturellen Kommunikation: Interagierende folgen nicht nur eigenen Gattungsregeln, sondern aufgrund bestimmten Vorwissens über das kommunikative Verhalten der anderen Kultur versuchen sie u. U., sich an den vermeintlichen Regeln der anderen Kulturgemeinschaft zu orientieren. Als Resultat haben wir hybride Texte, die weder den Konventionen der eigenen noch denen der fremden Kultur entsprechen. ${ }^{1}$

Die präsentierten Beispiele veranschaulichen unterschiedliche Aspekte kulturell variierender Gattungstraditionen und deren Folgen für die interkulturelle Kommunikation. Hierbei kommen nicht nur eigenkulturelle Gattungskonventionen (Formen, Funktionen und stilistische Bewertungen), die auf die interkulturelle Kommunikation übertragen werden, zum Vorschein, sondern auch Unsicherheiten - sofern die Interagierenden über eine bestimmte Gattung nicht verfügen. Darüberhinaus illustrieren die Beispiele auch die Eigendynamik interkultureller Begegnungen (Koole/ten Thije 1994) und den Einfluß bestimmter Erwartungshaltungen und bestimmten Vorwissens auf die kommunikative Praxis.

\section{Schlußfolgerungen}

Für die Forschung im Fach Deutsch als Fremdsprache (wie auch für die Interkulturelle Kommunikationsforschung) scheint mir die Untersuchung der Funktionen und Formen kommunikativer Gattungen - und damit verbunden die rhetorisch-stilistischen Konventionen der Durchführung bestimmter Textgattungen - von Relevanz. ${ }^{2}$ Sie sind - ähnlich wie Fix (1998: 18) im Zusammenhang mit schriftlichen Textsorten ausführt »Schnittstellen zwischen verschiedenen für sprachlich-kommunikatives Handeln relevanten Wissensbeständen, Komplexen von Wissenssystemen, zu denen neben Weltwissen [...], Kommunikationsnormenwissen und Sprachwissen auch Kulturwissen gehört«.

1 Vgl. auch Ehlich (1996: 928) zu »neuen sprachlichen Handlungsmustern«, die der interkulturellen Situation entstammen und »die in der (national)sprachlichen Homogenitätskonzeption von Kommunikation so von vorneherein nicht vorgesehen sind «.

2 Dies knüpft unmittelbar an die Forderung Ehlichs (1999) an, »alltägliche Wissenschaftssprache « zu untersuchen, wie beispielsweise die Praxis einer Vorlesung, einer Seminardiskussion, eines Laborgesprächs etc. 
Gerade Analysen »alltäglicher Wissenschaftssprache« (Ehlich 1999) - und damit verwoben Untersuchungen alltäglicher Wissenschaftsgattungen im Kulturvergleich, wie Vorlesungen, Laborgespräche, wissenschaftliche Vorträge und Diskussionen, Sprechstundengespräche etc. - sind ein Forschungsfeld, das mittels der Gattungsanalyse in Angriff genommen werden könnte. ${ }^{1}$

Bezüglich der Analyse von Textgattungen sind somit folgende Fragestellungen von Interesse:

1. Verfügen Sprecher/innen verschiedener Sprechgemeinschaften über vergleichbare Gattungen? Beispielsweise innerhalb der akademischen Gattungen wäre zu erforschen, inwiefern die Interagierenden über (mündliche und schriftliche) Gattungen wie Sprechstundengespräche, mündliche Prüfungsgespräche, Bewerbungsvorträge, Ausarbeitung und Präsentation von Thesen etc. verfügen.

2. Wie sieht die formale Gestaltung der betreffenden Gattung aus? Hierzu zählen textinterne Merkmale, Aspekte der interaktiven Realisierung wie auch textexterne Charakteristika der Außenstruktur der betreffenden Gattungen. In Zusammenhang mit wissenschaftlichen Vorträgen wäre u.a. zu fragen: Redet man frei, oder liest man ab? Beginnt man einen Vortrag eher mit einer persönlichen Anekdote oder mit einem
Sprichwort? Kritisiert man andere Positionen und Wissenschaftler eher direkt oder indirekt? Markiert man die Eigenund Fremdleistung deutlich, indem man Thesen anderer Wissenschaftler/ innen klar als solche abhebt? ${ }^{2}$ Wie signalisieren die Rezipienten/innen ihren Konsens bzw. Dissens? etc.

3. Haben vergleichbare Gattungen in den verschiedenen Sprechgemeinschaften vergleichbare interaktive Funktionen inne? (Beispielsweise: welche Funktionen haben Sprichwörter? Wie und in welchen Funktionen verwendet man Zitate?)

4. Existieren kulturell divergierende stilistische Bewertungen scheinbar gleicher Textgattungen?

5. Wie sind diese Differenzen in Zusammenhang mit kulturell vorhandenen kommunikativen Praktiken zu erklären? (Beispielsweise der Umgang mit Traditionalismus bzw. Individualismus; oder - wie Eßer (1997) in Zusammenhang mit geisteswissenschaftlichen Arbeiten mexikanischer Autor/ innen zeigt - die Verflechtung von Geisteswissenschaft und Literatur und damit einhergehend der ins Belletristische gehende Stil mexikanischer geisteswissenschaftlicher Texte.)

6. In welchen Kontexten und zu welchen Zwecken kann von den kanonisierten Formen abgewichen oder gar mit ihnen gespielt werden?

1 Erste Ansätze bieten u.a. Kotthoffs (1999) Untersuchung deutsch-russischer wissenschaftlicher Vorträge sowie Eßers (1997) Analyse deutsch-mexikanischer Unterschiede beim Abfassen geisteswissenschaftlicher studentischer Hausarbeiten. Letztere verdeutlicht eklatante Unterschiede zwischen den deutschen und mexikanischen Texttypen: Während sich die deutschen Texte durch »Theorielastigkeit, degressive Struktur und betont nüchterne, unpersönliche Darstellungsweise « auszeichnen, sind die mexikanischen Texte bestimmt durch »Konkretheit, koordinierende Themenentfaltung, subjektiv gefärbte Darstellungsweise und sprachliche Eleganz [...], die bisweilen ins Literarische geht « (Eßer 1997: 196 f.).

2 Vgl. Kotthoff (1999) speziell zu deutsch-russischen Differenzen sowie Roggausch (1998) zu kulturell divergierenden Textsortenregeln und Argumentationsstilen bei wissenschaftlichen Texten. 


\section{Literatur}

Auer, Peter: "Learning how to play the game: an investigation of role-played job interviews in East Germany«, Text 18 (1998), 7-38.

Bachtin, Michail M.: Die Ästhetik des Wortes. Frankfurt a. M.: Suhrkamp, 1979/86.

Baron, Bettina: »>Freiwillige Selbstkontrolle` im Fachgespräch. Selbstkritik und Skopuseinschränkung in Beiträgen von Wissenschaftlerinnen «, Zeitschrift für Germanistische Linguistik 139/140 (1998), 175-199.

Bausinger, Hermann: Formen der Volkspoesie. Berlin: Erich Schmidt, 1968.

Bergmann, Jörg: Klatsch. Zur Sozialform der diskreten Indiskretion. Berlin: de Gruyter, 1987.

Bergmann, Jörg; Luckmann, Thomas: »Reconstructive genres of everyday communication«. In: Quasthoff, Uta M. (ed.): Aspects of Oral Communication. Berlin: de Gruyter, 1995, 289-304.

Bourdieu, Pierre: Was heißt Sprechen? Die Ökonomie des sprachlichen Austausches. Wien: Braumüller, 1990.

Christmann, Gabriela B.: »Sich-Beklagen in Ökologiegruppen«. In: Bergmann, Jörg; Luckmann, Thomas (Hrsg.): Kommunikative Konstruktion von Moral. Band 1. Opladen: Westdeutscher Verlag, 1999, 151173.

Clyne, Michael: »Cultural differences in the organization of academic texts «, Journal of Pragmatics 11 (1987), 211-247.

Clyne, Michael: »Zu kulturellen Unterschieden in der Produktion und Wahrnehmung englischer und deutscher wissenschaftlicher Texte«, Info DaF 18 (1991), 457-474.

Ehlich, Konrad: »Interkulturelle Kommunikation «. In: Goebl, Hans; Nelde, Peter H.; Stary, Zdenek; Wölck, Wolfgang (Hrsg.): Kontaktlinguistik. Berlin: de Gruyter, 1996, 920-931.

Ehlich, Konrad: »Kritik der Wissenschaftssprachen «. In: Hoffmann, Lothar; Kalverkämper, Hartwig; Wiegand, Herbert Ernst (Hrsg.): Fachsprachen. Ein internationales Handbuch zur Fachsprachenforschung und Terminologiewissenschaft (HSK 14.1). New York; Berlin: de Gruyter, 1998, 856866.

Ehlich, Konrad: »Alltägliche Wissenschaftssprache«, Info DaF 26 (1999), 3-24. (Eben- falls abgedruckt in: Barkowski, Hans; Wolff, Armin (Hrsg.): Alternative Vermittlungsmethoden und Lernformen auf dem Prüfstand. Wissenschaftssprache - Fachsprache. Landeskunde aktuell. Interkulturelle Begegnung - Interkulturelles Lernen (Materialien Deutsch als Fremdsprache 52). Regensburg 1999, 1-30.

Ehlich, Konrad; Rehbein, Jochen: »Sprachliche Handlungsmuster «. In: Soeffner, Hans-Georg (Hrsg.): Interpretative Verfahren in den Sozial- und Textwissenschaften. Stuttgart: Metzler, 1970, 328-351.

Eßer, Ruth: >Etwas ist mir geheim geblieben am deutschen Referat<. Kulturelle Geprägtheit wissenschaftlicher Textproduktion und ihre Konsequenzen für den universitären Unterricht von Deutsch als Fremdsprache. München: iudicium, 1997.

Fix, Ulla: »Die erklärende Kraft von Textsorten. Textsortenbeschreibungen als $\mathrm{Zu}$ gang zu mehrfach strukturiertem - auch kulturellem - Wissen über Texte«, Linguistica XXX VII, 1 (1998), 15-27.

Gal, Susan: »Between Speech and Silence: The Problematics of Research on Language and Gender «, IPrA Papers in Pragmatics 3, 1 (1989), 271-289.

Galtung, Johan: »Struktur, Kultur und intellektueller Stil. Ein vergleichender Essay über sachsonische, teutonische, gallische und nipponische Wissenschaft «. In: Wierlacher, Alois (Hrsg.): Das Fremde und das Eigene. München: iudicium, 1985, 151193.

Gnutzmann, Claus: »>Abstracts< und > $\mathrm{Zu}$ sammenfassungen im deutsch-englischen Vergleich«. In: Müller-Jacquier, Bernd (Hrsg.): Interkulturelle Wirtschaftskommunikation. München: iudicium, 1991, 363-378.

Gumperz, John J.: Discourse strategies. Cambridge: Cambridge University Press, 1982.

Gülich, Elisabeth: »Was sein muß, muß sein. Überlegungen zum Gemeinplatz und seiner Verwendung «, Bielefelder Papiere zur Linguistik und Literaturwissenschaft 7 (1978), 1-41.

Günthner, Susanne: »Interkulturelle Aspekte von Schreibstilen: Zur Verwendung von Sprichwörtern und Routineformeln in Deutschaufsätzen chinesischer Deutschlerner/innen«. In: Lieber, Maria; Posset, Jürgen (Hrsg.): Texte Schreiben im 
Germanistik-Studium. München: iudicium, 1988, 145-159.

Günthner, Susanne: Diskursstrategien in der Interkulturellen Kommunikation. Analysen deutsch-chinesischer Gespräche. Tübingen: Niemeyer, 1993.

Günthner, Susanne: »Gattungen in der sozialen Praxis«, Deutsche Sprache 3 (1995), 193-217.

Günthner, Susanne: »Zur Aktualisierung kultureller Differenzen in Alltagsinteraktionen«. In: Rieger, Stefan; Schahadat, Schamma; Weinberg, Manfred (Hrsg.): Interkulturalität. Zwischen Inszenierung und Archiv. Tübingen: Narr, 1999, 251268.

Günthner, Susanne; Knoblauch, Hubert: ")Forms are the Food of Faith`. Gattungen als Muster kommunikativen Handelns «, Kölner Zeitschrift für Soziologie und Sozialpsychologie 4 (1994), 693-723.

Günthner, Susanne; Knoblauch, Hubert: »Culturally Patterned Speaking Practices - The Analysis of Communicative Genres«, Pragmatics 5, 1 (1995), 1-32.

Günthner, Susanne; Kotthoff, Helga: »Von fremden Stimmen. Weibliches und männliches Sprechen im Kulturvergleich«. In: Günthner, Susanne; Kotthoff, Helga (Hrsg.): Von fremden Stimmen: Weibliches und männliches Sprechen im Kulturvergleich. Frankfurt a. M.: Suhrkamp, 1991, 7-51.

Günthner, Susanne; Luckmann, Thomas: »Wissensasymmetrien in interkultureller Kommunikation«. Erscheint in: Apfelbaum, Birgit; Kotthoff, Helga (Hrsg.): Kultur im Gespräch. Opladen: Westdeutscher Verlag, 2001.

Günthner, Susanne; Luckmann, Thomas: "Asymmetries of Knowledge in Intercultural Communication: The Relevance of Cultural Repertoires of Communicative Genres«. Erscheint in: di Luzio, Aldo; Günthner, Susanne; Orletti, Franca (Hrsg.): Culture in Communication. Amsterdam: Benjamins, 2000.

Günthner, Susanne: Vorwurfsaktivitäten in der Alltagsinteraktion. Tübingen: Niemeyer, 2000.

Hahn, Alois: »Die soziale Konstruktion des Fremden«. In: Sprondel, Walter M. (Hrsg.): Die Objektivität der Ordnungen und ihre kommunikative Konstruktion. Frankfurt: Suhrkamp, 1994, 140-166.
Hanks, William F.: »Discourse Genres in a Theory of Practice«, American Ethnologist 14, 4 (1987), 668-692.

Kallmeyer, Werner: Kommunikation in der Stadt. Teil 1. Berlin; New York: de Gruyter, 1994.

Keim, Inken: »Formelhaftes Sprechen als konstitutives Merkmal sozialen Stils«. In: Sandig, Barbara; Selting, Margret (Hrsg.): Sprech- und Gesprächsstile. Berlin: de Gruyter, 1997, 318-344.

Kern, Frederike: „Culture, Genres, and the Problem of sequentidity«. Vortrag, gehalten auf der 7. International Pragmatics Conference, Budapest Juli 2000.

Koole, Tom; ten Thije, Jan D.: The construction of intercultural discourse. Amsterdam: Rodopi, 1994.

Kotthoff, Helga: »Der Tamada gibt bei Tisch den Ton an. Tafelsitten, Trinksprüche und Geschlechterrollen im kaukasischen Georgien «. In: Günthner, Susanne; Kotthoff, Helga (Hrsg.): Von fremden Stimmen. Frankfurt a.M.: Suhrkamp, 1991, 229260.

Kotthoff, Helga: »Disagreement and concession in disputes. On the context sensitivity of preference structures", Language in Society 22 (1993), 193-216.

Kotthoff, Helga: »Vortragsstile im Kulturvergleich: Zu einigen deutsch-russischen Unterschieden «. Vortrag, gehalten bei der Tagung PAS - Perspektiven auf Stil. Saarbrücken: Juni 1999.

Li, Xiangling: Chinese-Dutch Business Negotiations. Amsterdam: Rodopi, 1999.

Liang, Yong: "Zu soziokulturellen und textstrukturellen Besonderheiten wissenschaftlicher Rezensionen. Eine kontrastive Fachtextanalyse Deutsch/Chinesisch «, Deutsche Sprache 4 (1991), 289-311.

Lüger, Heinz-Helmut: Satzwertige Phraseologismen. Eine pragmalinguistische Untersuchung. Wien: Edition Praesens, 1999.

Luckmann, Thomas: »Kommunikative Gattungen im kommunikativen Haushalt einer Gesellschaft «. In: Smolka-Koerdt, Gisela et al. (Hrsg.): Der Ursprung der Literatur. München: Fink, 1988, 279-288.

Pomerantz, Anita: »Agreeing and disagreeing with assessments: some features of preferred/dispreferred turn shapes «. In: Atkinson, J.M.; Heritage, J. (Hrsg.): Structures of social action: Studies in conversation 
analysis. Cambridge: Cambridge University Press, 1984, 57-101.

Roggausch, Werner: »Kulturkontrast und Hermeneutik. Einige Notizen zur Begriffsbildung in den Geisteswissenschaften «, Jahrbuch Deutsch als Fremdsprache 24 (1998), 97-113.

Schütz, Alfred: »Der Fremde«. In: Schütz, Alfred (Hrsg.): Gesammelte Aufsätze. Den Haag: Martinus Nijhoff, 1972, 53-69.

Schwitalla, Johannes: „Sprachliche Ausdrucksformen für soziale Identität beim
Erzählen«. In: Kallmeyer, Werner (Hrsg.): Kommunikation in der Stadt. Berlin; New York: de Gruyter, 1994, 510-577.

Schwitalla, Johannes: Kommunikation in der Stadt. Teil 4. Berlin; New York: de Gruyter, 1995.

Swales, John M.: Genre Analysis. Cambridge: Cambridge University Press, 1990.

Vološinov, Valentin: Marxismus und Sprachphilosophie. Frankfurt a. M.: Ullstein, $1929 / 75$.

\section{Anhang: Transkriptionskonventionen}

\begin{tabular}{|c|c|}
\hline $\begin{array}{l}\text { [ja das] finde } \\
{[\mathrm{du} \mathrm{ab}]}\end{array}$ & $\begin{array}{l}\text { die innerhalb der Klammern stehenden Textstellen überlappen sich, } \\
\text { d. h. zwei Gesprächspartner reden gleichzeitig }\end{array}$ \\
\hline$()$. & kurzes Absetzen \\
\hline$(-)$ & Pause unter 0.5 Sek. \\
\hline$(0.5)$ & Pause von einer halben Sekunde \\
\hline$(.)$. & unverständlicher Text \\
\hline (gestern) & unsichere Transkription \\
\hline$=$ & direkter, schneller Anschluß zwischen zwei Äußerungen \\
\hline$=$ und $=$ dann $=$ ging $=$ & schnelles Sprechtempo \\
\hline$?$ & stark steigender Ton \\
\hline , & leicht steigender Ton \\
\hline . & fallender Ton \\
\hline , & schwebender Ton \\
\hline$\uparrow<$ Wort Wort $>$ & global hohe Tonhöhe \\
\hline$\downarrow<$ Wort Wort $>$ & global niedrige Tonhöhe \\
\hline$\uparrow$ wie & hoher Ansatz \\
\hline$\downarrow$ wie & tiefer Ansatz \\
\hline$\uparrow$ wo::hr $\downarrow$ & steigend-fallende Intonationskontur \\
\hline a: a:: & Silbenlängung \\
\hline${ }^{\circ}$ und so $^{\circ}$ & die Silben werden leiser gesprochen \\
\hline NEIN & die Silbe wird laut und betont gesprochen \\
\hline NEIN & die Silbe wird sehr laut und betont gesprochen \\
\hline mo((hi))mentan & die Äußerung wird kichernd gesprochen \\
\hline НАНАНА & lautes Lachen \\
\hline
\end{tabular}




\begin{tabular}{||l|l||}
\hline hihi & Kichern \\
\hline 'hh & starkes Ausatmen \\
\hline hh' & starkes Einatmen \\
\hline$<<$ hustet $>>$ & Kommentare (nonverbale Handlungen, o. ä.) \\
\hline
\end{tabular}

